\title{
Fratura por estresse diafisária do úmero em atleta de tênis adolescente: Relato de caso
}

\section{Humerus Diaphyseal Stress Fracture in a Teenage Tennis Athlete: Case Report}

\author{
Márcio Schiefer ${ }^{1,5}$ Francisco Nanci ${ }^{2 @}$ Luiz Eduardo Abílio ${ }^{3 \oplus}$ Yonder Archanjo Ching San Junior ${ }^{4}$ \\ Geraldo Motta Filho ${ }^{50}$
}

${ }^{1}$ Departamento de Ortopedia, Faculdade de Medicina, Universidade

Endereço para correspondência Márcio Schiefer, MD, MSc, PhD, Federal do Rio de Janeiro, Rio de Janeiro, RJ, Brasil Rua Nóbrega, 62/602, Niterói, RJ, 24220-320, Brasil

2 Hospital Central da Polícia Militar, Rio de Janeiro, RJ, Brasil (e-mail: marcioschiefer@hotmail.com).

${ }^{3}$ Estagiário de Ombro e Cotovelo, Instituto Nacional de

Traumatologia e Ortopedia, Rio de Janeiro, RJ, Brasil

${ }^{4}$ Universidade Federal do Estado do Rio de Janeiro, Rio de Janeiro, Brasil

${ }^{5}$ Centro de Cirurgia do Ombro e Cotovelo, Instituto Nacional de

Traumatologia e Ortopedia, Rio de Janeiro, RJ, Brasil

Rev Bras Ortop 2022;57(1):175-179.

\section{Resumo \\ Palavras-chave \\ - fraturas de estresse \\ - fraturas do úmero \\ - úmero \\ - tênis/lesões}

Um tenista adolescente tinha dor crônica no braço dominante durante os treinos de tênis. A ressonância magnética (RM) sugeriu lesão por estresse na diáfise do úmero. Depois de 4 semanas, ele se tornou assintomático e voltou a jogar. No entanto, houve recidiva da dor após 3 dias. A nova RM revelou fratura diafisária não desviada do úmero e edema significativo da medula óssea. O paciente ficou em repouso por 4 semanas. Depois disso, exercícios de fortalecimento foram introduzidos e o retorno aos treinamentos foi permitido após 12 semanas. Mesmo que assintomáticos, sugerimos que esses pacientes não voltem a jogar antes das 12 semanas, dependendo dos exames físicos e dos achados por imagem.

A teenage male tennis player had chronic pain in his dominant arm during tennis practice. Magnetic resonance imaging (MRI) suggested humerus diaphyseal stress injury. After 4 weeks, he became asymptomatic and resumed playing. However, pain recurred after 3 days. A new MRI revealed a diaphyseal undisplaced humerus fracture and significant bone marrow edema. The patient remained in rest for 4 weeks. After that, strengthening exercises were introduced and return to training was allowed after 12 weeks. Even if asymptomatic, we suggest that these patients should not return to play before 12 weeks, depending on the physical exam and imaging findings.

Este trabalho foi realizado no Instituto Nacional de Traumatologia e Ortopedia (INTO).

recebido

03 de Junho de 2020

aceito

17 de Setembro de 2020

Publicado on-line

Abril 19, 2021
DOI https://doi.org/

$10.1055 / \mathrm{s}-0040-1721837$. ISSN 0102-3616. (c) 2021. Sociedade Brasileira de Ortopedia e Traumatologia. All rights reserved.

This is an open access article published by Thieme under the terms of the Creative Commons Attribution-NonDerivative-NonCommercial-License, permitting copying and reproduction so long as the original work is given appropriate credit. Contents may not be used for commercial purposes, or adapted, remixed, transformed or built upon. (https://creativecommons.org/ licenses/by-nc-nd/4.0/)

Thieme Revinter Publicações Ltda., Rua do Matoso 170, Rio de Janeiro, RJ, CEP 20270-135, Brazil 


\section{Introdução}

Fraturas por estresse são lesões por sobrecarga comuns entre os atletas e representam um grande empecilho ao treinamento e às competições. ${ }^{1}$ Resultam de cargas excessivas, repetitivas e submáximas nos ossos, causando um desequilíbrio entre a reabsorção óssea e a formação. 0 microdano ósseo ocorre como resultado das atividades diárias e há reabsorção osteoclástica do osso danificado seguida pela produção óssea osteoblástica, resultando em um equilíbrio entre reabsorção e produção. ${ }^{2}$ Altas cargas de atividades físicas, pouco tempo para descansar, ingestão nutricional insuficiente ou uma combinação desses fatores podem levar a um desequilíbrio entre reabsorção e produção, com predominância da atividade osteoclástica. ${ }^{3}$ Fraturas por estresse nos membros superiores são muito menos comuns do que as dos membros inferiores e têm sido descritas em esportes dominados por membros superiores, como tênis ou as atividades de arremesso. ${ }^{4-6}$ Devido à sua baixa incidência, não há grandes séries publicadas na literatura, mas principalmente relatos de casos e séries de casos pequenos. ${ }^{1}$

Entre as fraturas por estresse nos membros superiores, as fraturas diafisárias do úmero são ainda mais raras. Rizzone et al. ${ }^{4}$ encontraram apenas 1 caso entre 671 fraturas por estresse em estudantes universitários. Changstrom et al. ${ }^{5}$ não encontraram nenhuma fratura diafisária no úmero entre 389 fraturas por estresse em atletas do ensino médio. Com base nos poucos relatos de casos disponíveis, pode-se inferir que essas fraturas predominam em duas faixas etárias principais: atletas adolescentes e atletas saudáveis de meiaidade. $^{7} \mathrm{O}$ alto nível de atividade, que coloca alto grau de estresse em um osso imaturo com desenvolvimento muscu- lar inadequado, explica as fraturas por estresse em atletas adolescentes. Embora a prevalência global de fraturas por estresse tenha se mostrado maior nas mulheres, ${ }^{4,5}$ parece que ambos os sexos são igualmente afetados em fraturas de estresse nos membros superiores, ${ }^{5}$ incluindo aquelas relacionadas à prática do tênis. ${ }^{3}$ Os sinais de exame físico incluem dor à palpação no local do estresse. ${ }^{7}$ A amplitude de movimento de ombro e cotovelo é tipicamente completa, mas a dor pode estar presente nas extremidades do movimento. ${ }^{7}$ A ressonância magnética (RM) é o principal exame para identificar alterações precoces de estresse no osso. ${ }^{2}$ Devido à escassez de casos disponíveis na literatura, não há consenso sobre como devemos tratar esses pacientes, especialmente no que diz respeito ao tempo que eles devem ficar afastados do treinamento.

\section{Relato de Caso}

Um tenista de 15 anos tinha dores crônicas e progressivas no braço direito, especialmente depois de treinos longos ou de competições. Um dia antes da consulta, no entanto, ele teve que interromper o treinamento devido à dor aguda intensa no braço. Exame físico revelou amplitude total de movimento e palpação dolorosa dos terços médio e distal do braço (-Fig. 1). A RM revelou extensa reação de estresse na diáfise umeral (-Fig. 2). O paciente interrompeu o treinamento e iniciou a fisioterapia, com resolução completa da dor após alguns dias. Após 4 semanas, ele permaneceu assintomático e uma nova RM mostrou significativa regressão do edema ósseo. Apesar das recomendações médicas de retorno progressivo ao esporte com cargas reduzidas de treinamento, ele começou os treinamentos pesados para participar de sua próxima competição

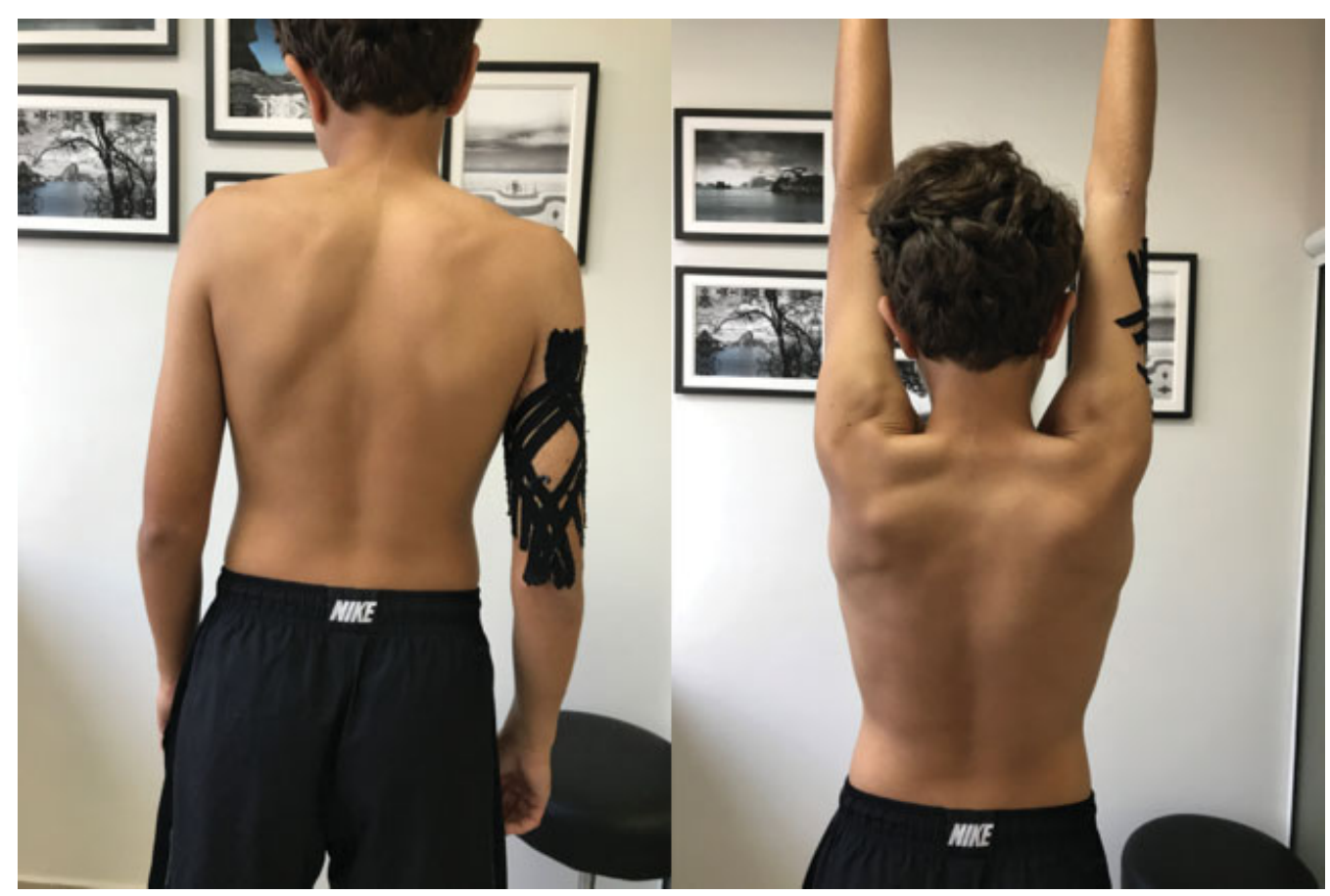

Fig. 1 Aspecto clínico do paciente, mostrando elevação total do ombro. 

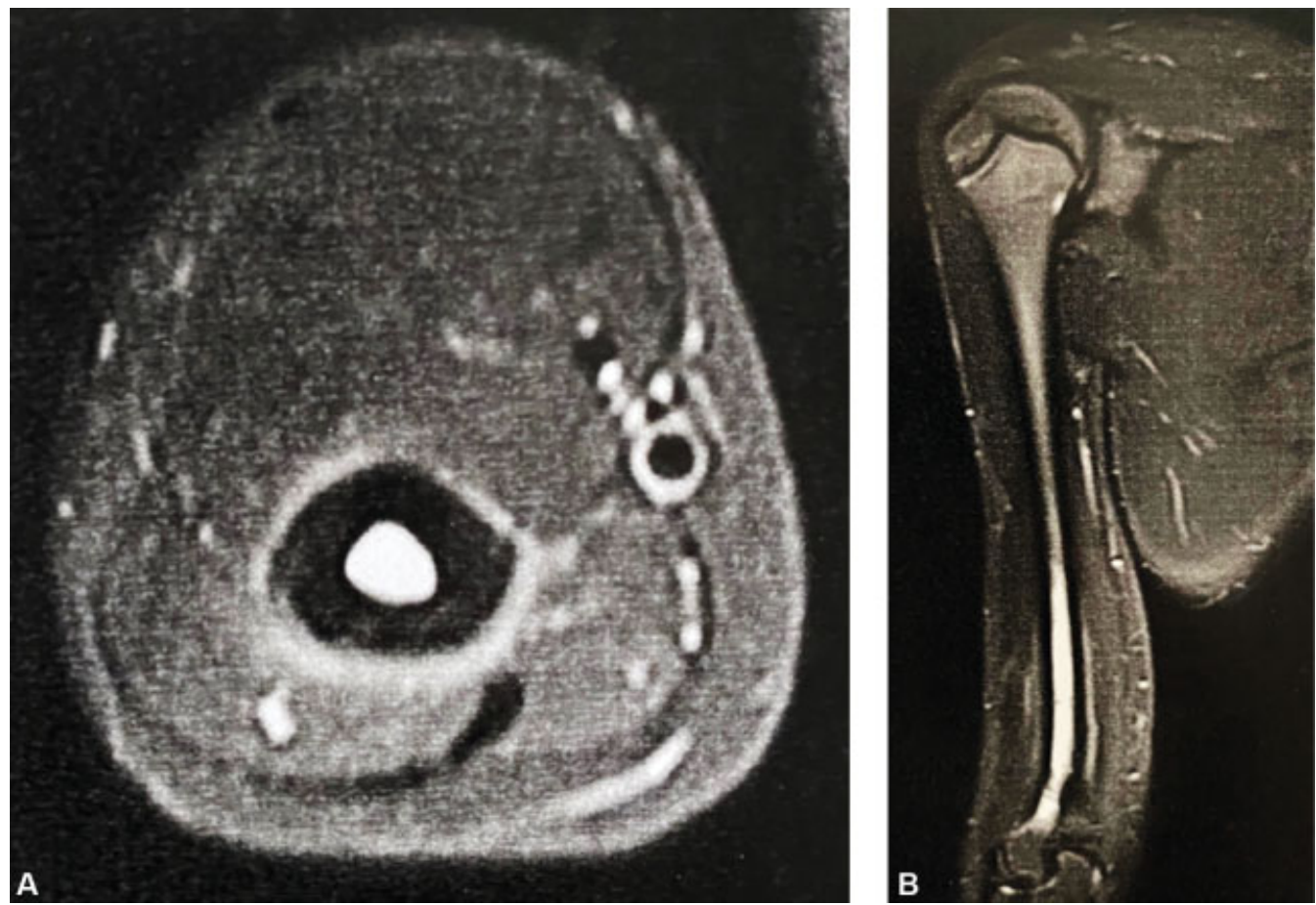

Fig. 2 Ressonância magnética ponderada por T2 axiais (A) e coronal (B) com peso de gordura, mostrando edema significativo de medula óssea e edema periosteal na metade inferior da diáfise do úmero.

programada. Após 3 dias de treinamento intensivo, a dor recorreu. $\mathrm{O}$ treinamento foi então interrompido e o paciente retornou à fisioterapia. A nova ressonância magnética detectou uma fratura por estresse não deslocada na diáfise do úmero direito (-Fig.3). Após um período de poucos dias, o alívio total da dor foi alcançado mais uma vez, mas o atleta só foi autorizado a realizar exercícios de membros inferiores e core. Um mês depois, a nova RM revelou regressão do edema ósseo (-Fig. 4). Foi introduzido o fortalecimento muscular, focado em bíceps e tríceps e evitando movimentos rotacionais do braço. Além disso, nenhum trabalho com raquete foi permitido. Após mais 1 mês, tomografia computadorizada mostrou formação de calo periosteal (-Fig. 5). Exercícios de fortalecimento muscular foram intensificados e treinamento
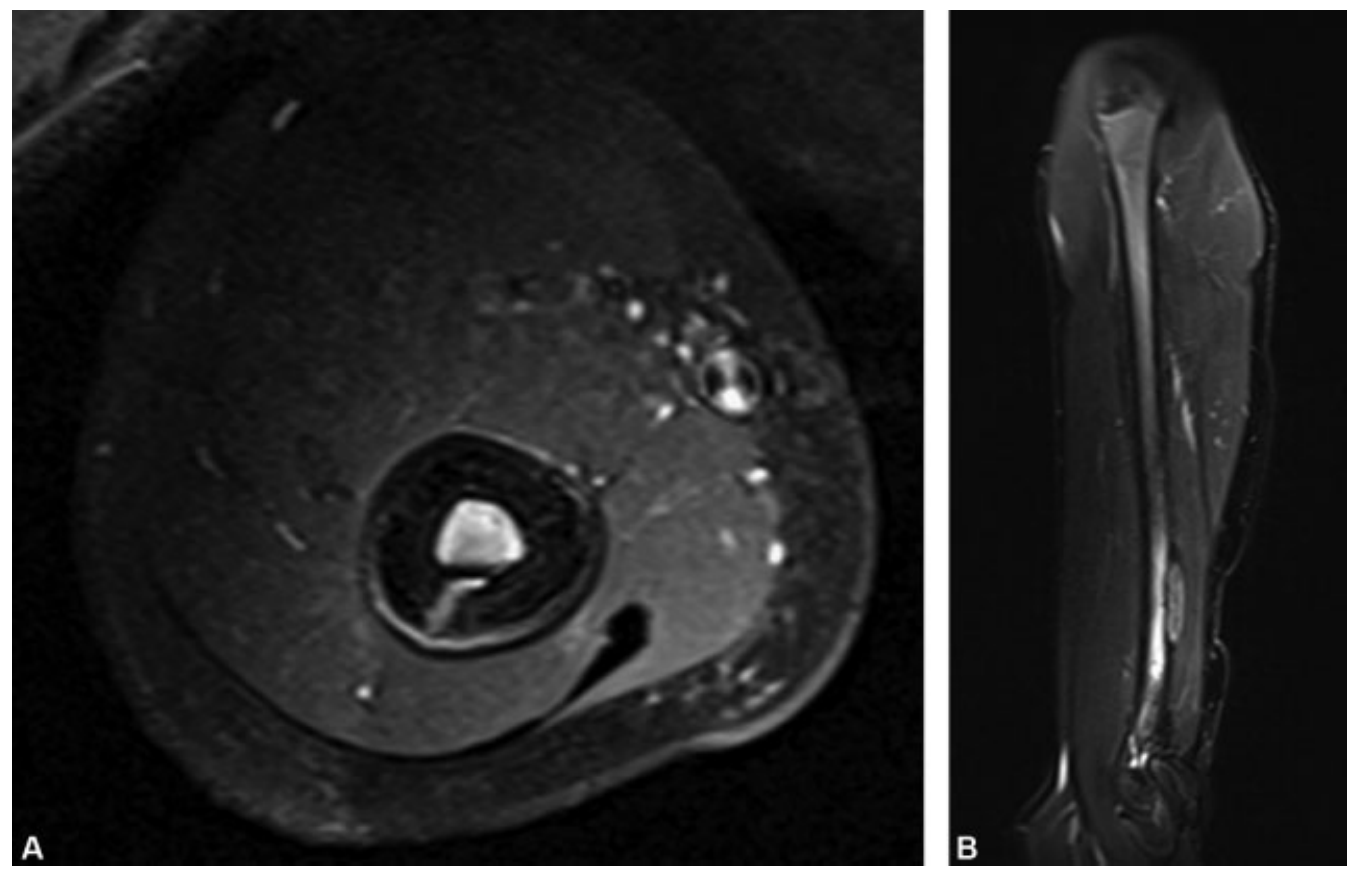

Fig. 3 (A) Imagem axial STIR de ressonância magnética. revelando fratura longitudinal através do córtex posterior, que é espessa; edema endosteal e periosteal também são notados. (B) Imagem sagital STIR de RM mostrando espessamento cortical e edema de medula óssea em diáfise do úmero distal. 

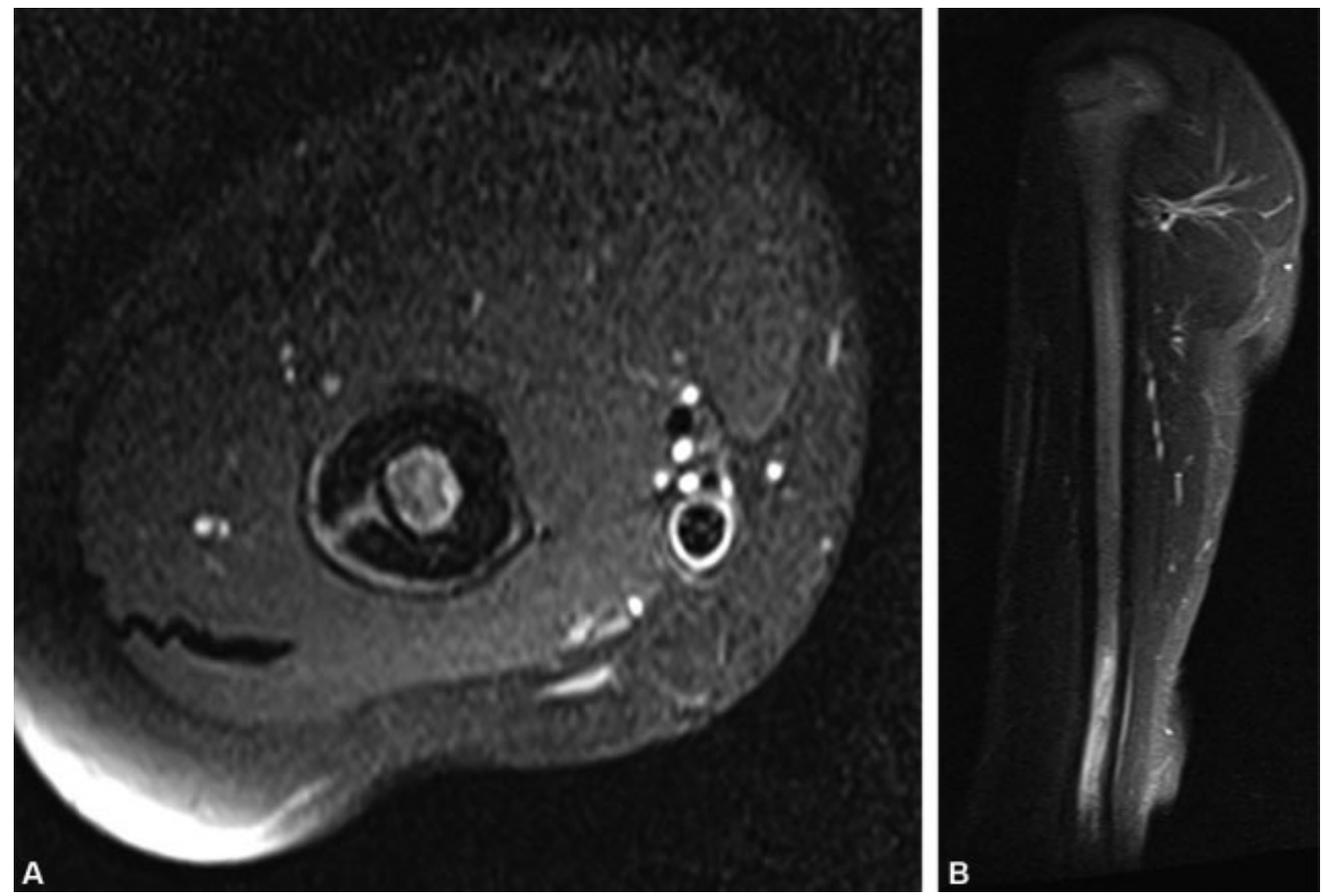

Fig. 4 Imagens de próton axial, suprimidas por gordura (A) e STIR sagital (B) imagens de ressonância magnética que retratam a manutenção do espessamento do córtex posterior, linha de fratura linear e ligeira redução no edema periosteal e endosteal.
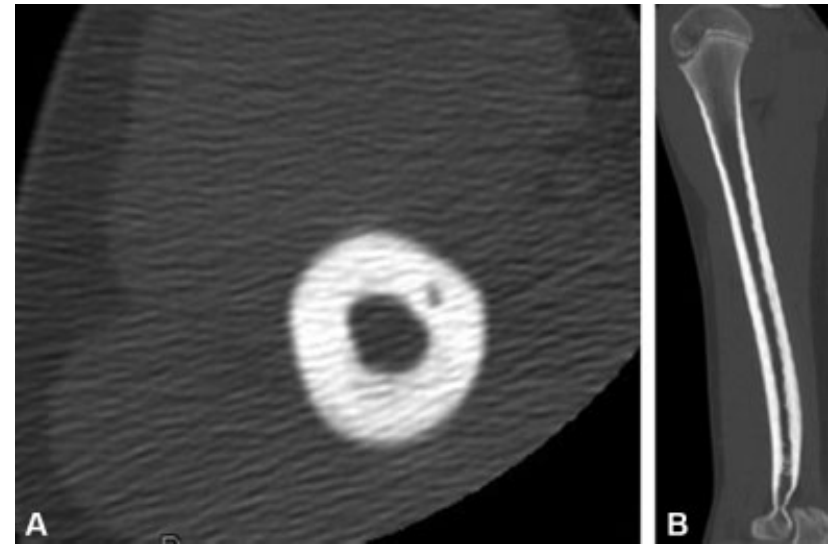

Fig. 5 Imagens axiais (A) e sagital CT (B) mostrando consolidação da fratura e espessamento persistente do córtex posterior.

específico do gestual desportivo do tênis foi introduzido. Dez semanas após a recorrência da dor, o paciente foi orientado a retomar o treinamento usando bolas macias e uma raquete mais leve do que a habitual. Duas semanas depois, uma nova RM confirmou consolidação completa e edema ósseo residual (-Fig. 6), para que o atleta voltasse a treinar com bolas e raquetes habituais. As cargas de treinamento foram progressivamente aumentadas e o atleta pôde competir 2 meses depois. Ele permanece assintomático após um período de 3 anos de seguimento.

\section{Discussão}

Fraturas por estresse não são comuns em tenistas. Iwamoto et al. ${ }^{8}$ estudaram 196 casos de fraturas por estresse em mais de 10.000 atletas e descobriram que apenas $2,6 \%$ de todas as

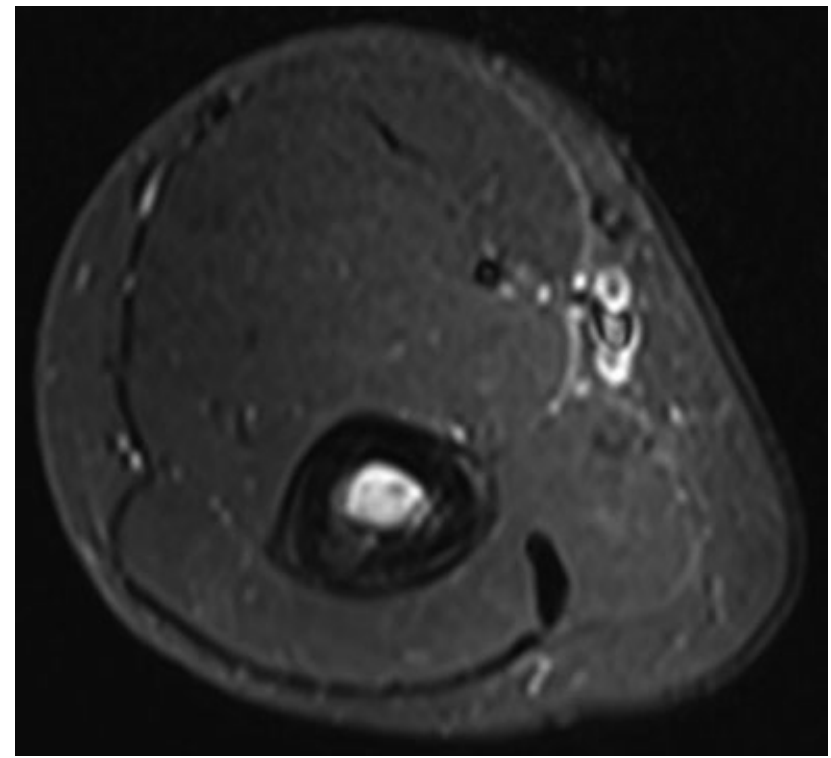

Fig. 6 Imagem de ressonância magnética do braço, no plano axial, ponderada em densidade protônica com supressão de gordura, revelando edema residual de medula óssea. A linha de fratura não é mais evidente.

fraturas ocorreram em tenistas. Eles também observaram que apenas $1,4 \%$ de todos os tenistas sintomáticos sofreram fraturas por estresse, nenhuma delas nos membros superiores. Maquirriain et al. ${ }^{3}$ encontraram maior incidência de lesões por estresse em tenistas de elite, compreendendo 12,9\% dos 139 indivíduos. A maior taxa relatada em seu artigo pode ser devido à inclusão de todas as lesões por estresse, em vez de apenas fraturas por estresse. Embora tenham mostrado que as lesões por estresse nos membros superiores representaram 
$22 \%$ de todos os ferimentos, nenhuma ocorreu no úmero. Rizzone et al. ${ }^{4}$ avaliaram 671 atletas universitários com quase 12.000 exposições-atletas (um atleta participando de um treino ou competição) e encontraram apenas $1,2 \%$ de todas as fraturas por estresse ocorridas em tenistas, nenhuma delas no membro superior.

Atletas adolescentes parecem estar em maior risco de fraturas por estresse. Maquirriain et al. ${ }^{3}$ encontraram uma incidência estatisticamente maior de fraturas por estresse em tenistas de elite júnior do que em jogadores profissionais (20,3 versus $7,5 \%$ ). Milgrom et al. ${ }^{9}$ relatou que a cada ano de aumento na idade de 17 para 26 anos, o risco de fratura por estresse em qualquer local diminuiu 28\%. Tenistas competitivos adolescentes geralmente se envolvem em participação pesada e profissional e têm longas sessões diárias de treinamento desde cedo. Muitos deles são submetidos ao volume de treinamento adulto, para o qual não estão preparados. O estirão puberal leva ao rápido crescimento ósseo, mas os músculos não se desenvolvem tão rápido. ${ }^{7}$ Acredita-se que o estresse torcional seja o principal mecanismo de fraturas por estresse nos tenistas, ${ }^{3}$ pois a poderosa ação rotacional transmite força considerável através dos ossos do membro superior. Foi sugerido que as forças compressivas dos bíceps e tríceps através do eixo umeral são protetoras contra as forças rotacionais durante a prática do tênis. ${ }^{7}$ Quando esses músculos fadigam, sua capacidade de dissipar energia reduz e uma maior tensão rotacional ocorre no úmero, permitindo que ocorram lesões por estresse. Portanto, exercícios de fortalecimento muscular também podem ser importantes na prevenção dessas lesões. O descanso parece ser outro fator protetor chave. Foi sugerido que todos os adolescentes envolvidos no tênis competitivo devem descansar pelo menos 1 dia por semana e realizar 1 semana de treinamento leve por mês. ${ }^{3}$

Tenistas de elite com lesões por estresse no úmero podem sofrer lesões concomitantes no mesmo membro. Hoy et al. ${ }^{10}$ descobriram que seis dos oito atletas com lesões por estresse no úmero tiveram lesões recentes ou simultâneas no ombro. Os autores trabalham com a hipótese de que esses atletas podem impor alto estresse no osso como resultado de compensação por uma lesão ipsilateral. Para manter o poder do golpe ou para transmitir um grau de giro na bola, um jogador pode usar demais regiões sãs do membro para compensar a falta de geração de energia mais proximal. ${ }^{10}$

0 tratamento de fraturas por estresse em atletas representa um desafio, mesmo em relação àquelas mais comuns. Em primeiro lugar, os atletas são particularmente resistentes a interromper a prática esportiva e, uma vez que obtêm melhora da dor, mesmo que parcial, querem retomar os treinos e competir. Em segundo lugar, o compromisso anterior com torneios ou campeonatos é outro motivo para o atleta acelerar o retorno ao jogo. Em terceiro lugar, alguns atletas tendem a voltar ao esporte com cargas excessivamente altas de treinamento, e isso pode predispor à recorrência de lesões. De fato, Rizzone et al. ${ }^{4}$ mostraram que quase um quarto das fraturas por estresse eram recorrentes em sua série. O tratamento das fraturas por estresse do úmero parece ser ainda mais desafiador devido à escassez de dados na literatura e aos poucos casos relatados. A falta de um protocolo de tratamento significa que cada atleta tem que ser tratado individualmente. No caso do relatório, o paciente continuou jogando por meses, apesar de ter dor. Uma vez que o diagnóstico de reação de estresse foi feito, ele parou de treinar e tornou-se assintomático logo depois. Quatro semanas depois, a nova RM mostrou regressão do edema ósseo e o paciente retomou o treinamento. No entanto, ele treinou muito duro e a dor voltou logo após 3 dias. Uma nova RM revelou uma fratura por estresse e o paciente teve que ficar ainda mais tempo afastado da prática esportiva. Depois que a fratura por estresse foi detectada, o paciente permaneceu 4 semanas em repouso, mais 4 semanas fazendo exercícios de fortalecimento, e treinamento leve só foi permitido após 12 semanas.

Com base na experiência com este caso, recomendamos uma abordagem mais conservadora no tratamento de atletas que sofrem fraturas diafisárias por estresse do úmero, com repouso, fisioterapia, fortalecimento muscular e progressão gradual da carga de treinamento. Em nossa opinião, o retorno ao jogo deve ser adiado até 12 semanas, mesmo que o paciente se torne assintomático antes desse tempo.

\section{Suporte Financeiro}

Não houve apoio financeiro de fontes públicas, comerciais, ou sem fins lucrativos.

Conflito de Interesses

Os autores não têm conflito de interesses a declarar.

\section{Referências}

1 Smith SR, Patel NK, White AE, Hadley CJ, Dodson CC. Stress Fractures of the Elbow in the Throwing Athlete: A Systematic Review. Orthop J Sports Med 2018;6(10):2325967118799262

2 Anderson MW. Imaging of upper extremity stress fractures in the athlete. Clin Sports Med 2006;25(03):489-504, vii

3 Maquirriain J, Ghisi JP. The incidence and distribution of stress fractures in elite tennis players. Br J Sports Med 2006;40(05): 454-459, discussion 459

4 Rizzone KH, Ackerman KE, Roos KG, Dompier TP, Kerr ZY. The epidemiology of stress fractures in collegiate student-athletes, 2004-2005 through 2013-2014 academic years. J Athl Train 2017;52(10):966-975

5 Changstrom BG, Brou L, Khodaee M, Braund C, Comstock RD. Epidemiology of stress fracture injuries among US high school athletes, 2005-2006 through 2012-2013. Am J Sports Med 2015; 43(01):26-33

6 Gregory P. Upper extremity injuries in the athlete. J Orthop Trauma 1995;9(04):361

7 Jones GL. Upper extremity stress fractures. Clin Sports Med 2006; 25(01):159-174, xi

8 Iwamoto J, Takeda T. Stress fractures in athletes: review of 196 cases. J Orthop Sci 2003;8(03):273-278

9 Milgrom C, Finestone A, Shlamkovitch N, et al. Youth is a risk factor for stress fracture. A study of 783 infantry recruits. J Bone Joint Surg Br 1994;76(01):20-22

10 Hoy G, Wood T, Phillips N, Connell D, Hughes DC. When physiology becomes pathology: the role of magnetic resonance imaging in evaluating bone marrow oedema in the humerus in elite tennis players with an upper limb pain syndrome. Br J Sports Med 2006; 40(08):710-713, discussion 713 